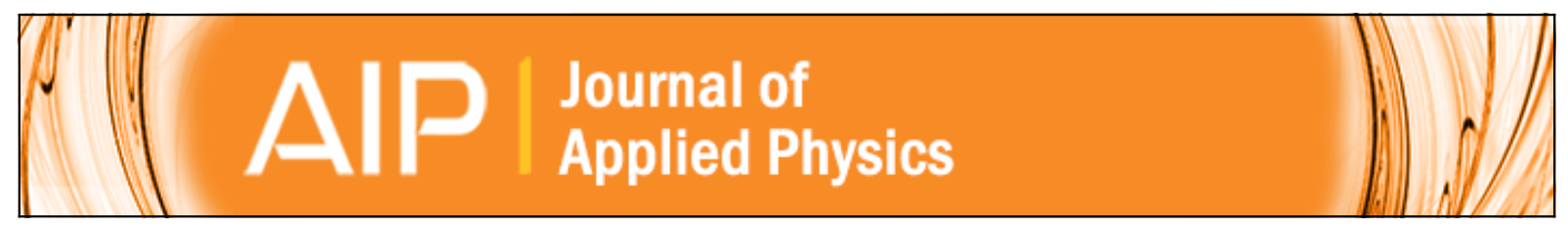

\title{
Comparison of noise floor and sensitivity for different magnetoelectric laminates
}

Junqi Gao, Jaydip Das, Zengping Xing, Jiefang Li, and D. Viehland

Citation: Journal of Applied Physics 108, 084509 (2010); doi: 10.1063/1.3486483

View online: http://dx.doi.org/10.1063/1.3486483

View Table of Contents: http://scitation.aip.org/content/aip/journal/jap/108/8?ver=pdfcov

Published by the AIP Publishing

\section{Articles you may be interested in}

Enhancement in magnetic field sensitivity and reduction in equivalent magnetic noise by magnetoelectric laminate stacks

J. Appl. Phys. 111, 104504 (2012); 10.1063/1.4718441

Enhanced sensitivity to direct current magnetic field changes in Metglas/Pb(Mg1/3Nb2/3)O3-PbTiO3 laminates J. Appl. Phys. 109, 074507 (2011); 10.1063/1.3569629

Ring-type electric current sensor based on ring-shaped magnetoelectric laminate of epoxy-bonded Tb 0.3 Dy 0.7 $\mathrm{Fe} 1.92$ short-fiber/NdFeB magnet magnetostrictive composite and $\mathrm{Pb}(\mathrm{Zr}$, Ti)O 3 piezoelectric ceramic J. Appl. Phys. 107, 09D918 (2010); 10.1063/1.3360349

Circumferential-mode, quasi-ring-type, magnetoelectric laminate composite-a highly sensitive electric current andor vortex magnetic field sensor

Appl. Phys. Lett. 86, 182506 (2005); 10.1063/1.1923184

Extremely low frequency response of magnetoelectric multilayer composites Appl. Phys. Lett. 86, 102901 (2005); 10.1063/1.1881784

MIT LINCOLN LABORATORY CAREERS

Discover the satisfaction of innovation and service to the nation
- Space Control

- Air \& Missile Defense

- Communications Systems \& Cyber Security

- Intelligence, Surveillance and

Reconnaissance Systems
- Advanced Electronics

- Tactical Systems

- Homeland

Protection

- Air Traffic Control

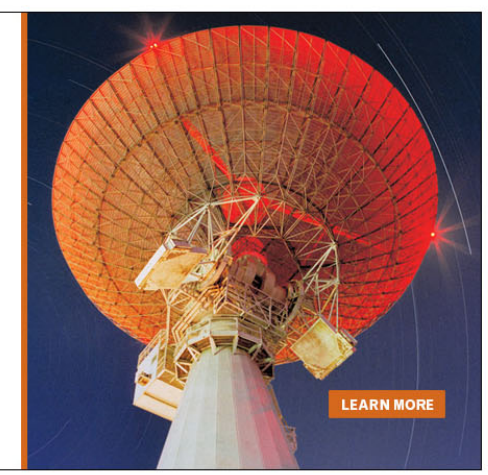




\title{
Comparison of noise floor and sensitivity for different magnetoelectric laminates
}

\author{
Junqi Gao, ${ }^{\text {a) }}$ Jaydip Das, Zengping Xing, Jiefang Li, and D. Viehland \\ Department of Materials Science and Engineering, Virginia Tech, Blacksburg, Virginia 24061, USA
}

(Received 16 May 2010; accepted 7 August 2010; published online 25 October 2010)

\begin{abstract}
We present a comparison of the magnetoelectric (ME) response and magnetic-field sensitivities of engineered laminate sensors comprised of magnetostrictive and piezoelectric phases. The ME voltage coefficients for Metglas and single crystal fibers of $\mathrm{Pb}\left(\mathrm{Mg}_{1 / 3} \mathrm{Nb}_{2 / 3}\right) \mathrm{O}_{3}-\mathrm{PbTiO}_{3}(\mathrm{PMN}-\mathrm{PT})$ or $\mathrm{Pb}\left(\mathrm{Zn}_{1 / 3} \mathrm{Nb}_{2 / 3}\right) \mathrm{O}_{3}-\mathrm{PbTiO}_{3}$ (PZN-PT) are about 2.8 times larger than those with Metglas- $\mathrm{Pb}(\mathrm{Zr}, \mathrm{Ti}) \mathrm{O}_{3}(\mathrm{PZT})$ ceramic ones. This results in a 1.7 times enhancement in the magnetic-field sensitivity for the structures with single crystals. Accordingly, the noise floors are about three to four times lower for composites with PMN-PT or PZN-PT fibers than those with PZT. (C) 2010 American Institute of Physics. [doi:10.1063/1.3486483]
\end{abstract}

\section{INTRODUCTION}

Magnetoelectric (ME) materials have been of recent research interest due to their potential for sensor, data storage, and communication applications. ${ }^{1}$ These materials are capable of exhibiting a change in polarization with applied magnetic field, or a change in magnetization with applied electric field. The ME effect was first observed about 50 years ago in $\mathrm{Cr}_{2} \mathrm{O}_{3}$ single crystal, which had a small $\mathrm{ME}$ voltage coefficient of $\alpha_{\mathrm{ME}} \approx 20 \mathrm{mV} / \mathrm{cm} \mathrm{Oe}$. $^{2}$ However, the scarcity of single phase materials with high ME coupling at room temperature has stimulated research focused on the development of engineered ME layered ${ }^{3}$ and nanopillar-embedded-in-matrix ${ }^{4}$ heterostructures.

The engineered ME structures that have high ME responses are two phase composites consisting of magnetostrictive and piezoelectric layers. ${ }^{5,6}$ The ME coupling mechanism in these multiphase structures occurs through a transformation of a magnetic/electric field induced strain from the magnetic/electric phase to the adjacent electric/ magnetic phase, via a magnetoelastoelectric interaction. The value of the ME voltage coefficient for these heterostructures is about several orders of magnitude higher than that for the single phase materials: for example, long sandwiched laminate structures comprised of magnetostrictive Metglas and piezoelectric $\mathrm{Pb}(\mathrm{Zr}, \mathrm{Ti}) \mathrm{O}_{3}$ or PZT layers have $\alpha_{\mathrm{ME}}$ $\leq 22 \mathrm{~V} / \mathrm{cm} \mathrm{Oe}^{7}$ Such a high ME response offers the potential to tune electrical responses with small applied magnetic fields, and to detect small magnetic field variations using a simple operational amplifier. ${ }^{8,9}$ Accordingly, magnetostrictive-piezoelectric laminate composites have the potential to be a new class of magnetic-field sensors that are small, room-temperature operational, cost-effective, and low power consuming.

There have been a number of studies focused on the optimization of ME coupling in these heterostructures. Structures comprised of various piezoelectric phases with high piezoelectric $d_{33}$ and electromechanical coupling $k_{33}$ coeffi-

${ }^{a)}$ Electronic mail: junqi08@vt.edu. cients [such as $\mathrm{PZT}, \mathrm{Pb}\left(\mathrm{Mg}_{1 / 3} \mathrm{Nb}_{2 / 3}\right) \mathrm{O}_{3}-\mathrm{PbTiO}_{3}$ (PMN-PT) and $\left.\mathrm{Pb}\left(\mathrm{Zn}_{1 / 3} \mathrm{Nb}_{2 / 3}\right) \mathrm{O}_{3}-\mathrm{PbTiO}_{3}(\mathrm{PZN}-\mathrm{PT})\right]$ and various magnetostrictive phases with high piezomagnetic coefficients (such as Terfenol-D, Metglas) have been investigated. ${ }^{6-10}$ Furthermore, different operational modes (such as longitudinal-longitudinal or L-L, and push-pull) were also studied. ${ }^{6}$ To date, laminate composites consisting of Metglas and PZT fiber ${ }^{5}$ with a push-pull configuration have been found to have the highest ME coefficient. However, this laminate configuration has not yet been optimized with regards to the piezoelectric phase.

In addition, noise power spectra and the present dominant noise source have not yet been determined for the ME composites. This is a very important set of information for practical applications, as it is expected to provide insight into the sensor performance. Prior investigations on the noise floor of other types of magnetic sensors are well-known. These include giant magnetoresistive, flux-gate, and superconducting quantum interference devices which have noise floors in a magnetically shielded environment on the order of $10^{-10} \mathrm{~T} / \sqrt{\mathrm{Hz}}, \quad 10^{-12} \mathrm{~T} / \sqrt{\mathrm{Hz}}$, and $10^{-14} \mathrm{~T} / \sqrt{\mathrm{Hz}}$, respectively, near frequencies of about $1<f<10^{3} \mathrm{~Hz} .{ }^{11-13}$

Here, we report the development of ME laminate layered structures with magnetostrictive Metglas and piezoelectric PMN-PT and PZN-PT single crystal fibers. The ME voltage coefficient shows an increase in about 2.8 times relative to that previously reported for Metglas-PZT structures of similar size. This results in a 1.7 times increase in the magneticfield sensitivity and a decrease in the noise floor of about three to four times (to about $20 \mathrm{pT} / \sqrt{\mathrm{Hz}}$ in the frequency range $f=10-10^{3} \mathrm{~Hz}$ ) relative to laminates with PZT ceramic fibers.

\section{EXPERIMENTAL DETAILS}

To fabricate ME sensors, we obtained PZT (CTS, Albuquerque, NM), PMN-PT single crystals (Shanghai Institute of Ceramics, Shanghai, China), PZN-PT single crystals (Microfine Materials Technologies Pte Ltd., Singapore), and Metglas (Metglas Inc., Anderson, SC). Piezoelectric fibers of $200 \mu \mathrm{m}$ thickness were then cut to the dimensions of 2.5 


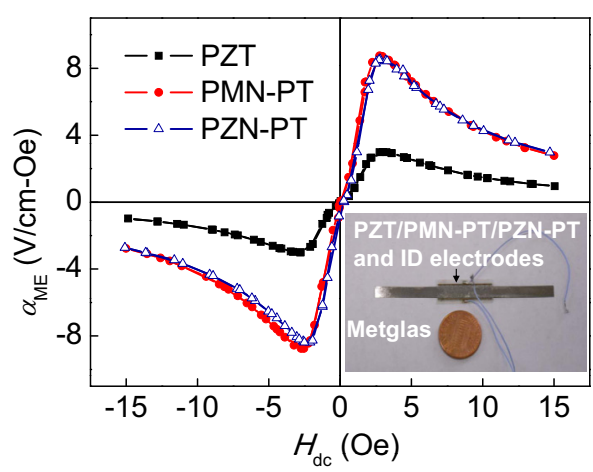

FIG. 1. (Color online) The ME voltage coefficient $\alpha_{\mathrm{ME}}$ as a function of the static magnetic field $H_{\mathrm{dc}}$ for Metglas-PZT, Metglas-PMN-PT, and MetglasPZN-PT laminate composites, as indicated. Inset shows a representative picture of a laminate composite.

$\times 0.4 \mathrm{~cm}^{2}$, and both surfaces of the fibers were adhered to thin polymer insulating films with interdigitated electrodes using an epoxy resin. This electrode pattern allowed us to symmetrically pole the piezoelectric fibers in a back-to-back pattern along their length axis. ${ }^{9}$ Next, these structures were laminated together between four Metglas layers of dimension $8 \times 0.4 \mathrm{~cm}^{2}$ using an epoxy. The thickness of each Metglas layer was $25 \mu \mathrm{m} .{ }^{14}$ Low noise operational amplifier detection circuits were fabricated following recent reports. ${ }^{15} \mathrm{We}$ then assembled the ME sensors and circuits into battery operated sensor detection units. The detection units were designed to operate over the bandwidth of $1<f<10^{3} \mathrm{~Hz}$. The output of the ME sensor unit was connected to a spectrum analyzer (SR-785) to measure the noise floor in this frequency range.

\section{RESULTS AND DISCUSSION}

The ME coefficient $\alpha_{\mathrm{ME}}$ was first measured as a function of dc magnetic field $H_{\mathrm{dc}}$ for various laminates using a lock-in amplifier method. A pair of Helmholtz coils was used to generate an ac magnetic field of $H_{\mathrm{ac}}=1$ Oe at a frequency of $f=1 \mathrm{kHz}$. The $H_{\mathrm{dc}}$ was applied along the longitudinal axis of the laminates. Figure 1 shows $\alpha_{\mathrm{ME}}$ as a function of $H_{\mathrm{dc}}$ for Metglas-PZT, Metglas-PMN-PT, and Metglas-PZN-PT laminates. The inset shows a representative photo of one of our laminate structures. From Fig. 1, we can see that for all three laminates the $\alpha_{\mathrm{ME}}$ increases with increasing dc magnetic bias up to about $H_{\mathrm{dc}}=3$ Oe, reaches a maximum, and subsequently decreases as $H_{\mathrm{dc}}$ increases further. The values of $\alpha_{\mathrm{ME}}$ for the Metglas-PMN-PT and Metglas-PZN-PT fiber laminates are nearly equal and both are notably higher than that for Metglas-PZT. The maximum value of $\alpha_{\mathrm{ME}}$ for PMN-PT and PZN-PT based laminates is about $8.5 \mathrm{~V} / \mathrm{cm}$ Oe, which is about 2.8 times larger than that for the PZT based ones of similar size. This increase in $\alpha_{\mathrm{ME}}$ can be attributed to the higher piezoelectric coefficients for PMN-PT and PZN-PT $\left(d_{33} \cong 1700 \mathrm{pC} / \mathrm{N}\right)$ relative to that of PZT $\left(d_{33}\right.$ $\cong 400 \mathrm{pC} / \mathrm{N}$ ), resulting in a larger change in induced polarization under magnetic fields.

The ac magnetic-field sensitivity was measured next using a low noise detection circuit. ${ }^{15}$ A small ac magnetic field was applied along the longitudinal direction of the laminate

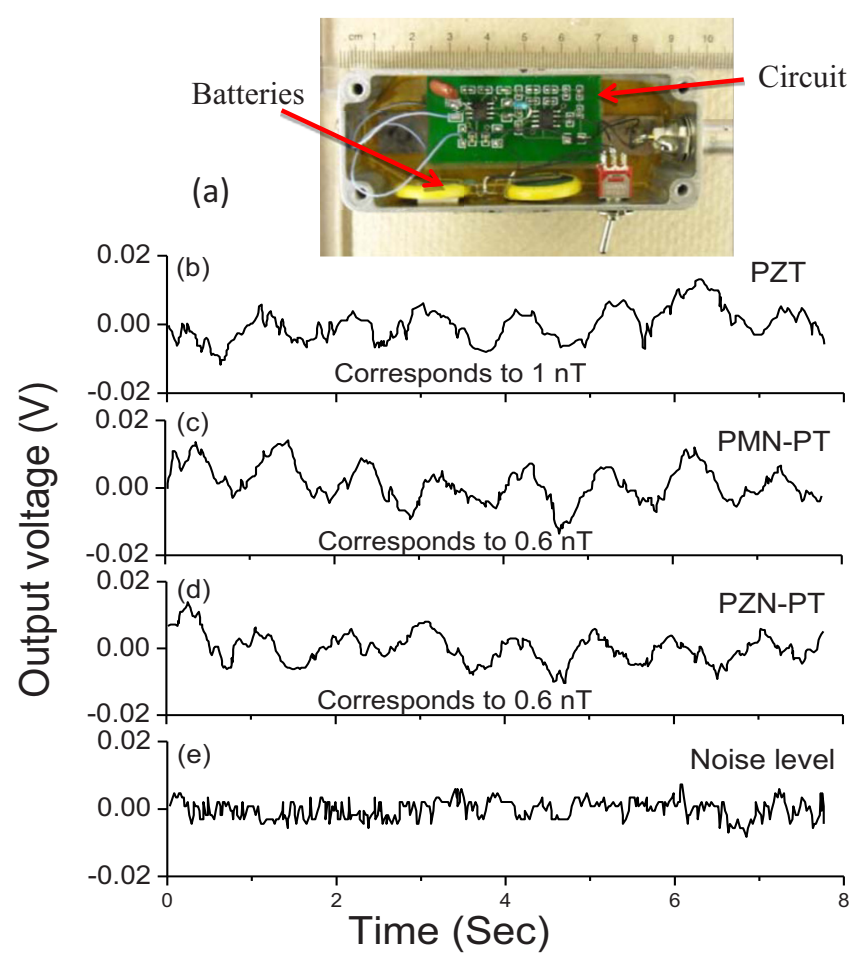

FIG. 2. (Color online) (a) Picture of our low noise circuit along with the ME sensor in a box. The ME output voltage as a function of time for the (b) PZT, (c) PMN-PT, and (d) PZN-PT laminates with low noise circuit, respectively. The corresponding field sensitivities are as indicated. (e) Noise level for our detection unit.

by inputting an ac signal of frequency $f=1 \mathrm{~Hz}$ into the Helmholtz coils. This ac signal was generated and monitored by a lock-in amplifier (SR-850). The details of the ac magnetic-field calibration have previously been reported. ${ }^{9}$ In order to bias the ME laminate to the highest value of $\alpha_{\mathrm{ME}}$ near the inflection point in the $\varepsilon-H$ curve, small permanent magnets were attached to the laminates. The output voltage from the laminates and the noise levels of the detection units were measured in the time domain using an oscilloscope (Agilent 54624A). Details of the measurement can be found in Ref. 9. The peak-to-peak voltage noise level was about 6 $\mathrm{mV}$. The applied ac magnetic field was varied to keep the peak-to-peak output voltage constant at about $12 \mathrm{mV}$ where the signal to noise ratio was equal to two: this was done in order to compare the magnetic-field sensitivity for different laminates under the same conditions.

Figure 2 shows the magnetic-field sensitivity results. Panel (a) shows a photo of the detection circuit. This box contains the ME laminate, the low noise detection circuit, and several batteries that served as the power supply for the circuit. Panels (b), (c), and (d) show the time domain output waveforms for the Metglas-PZT, Metglas-PMN-PT, and Metglas-PZN-PT laminates, respectively. The corresponding magnetic-field sensitivities are also provided in the graphs. The magnetic-field sensitivities are $0.6 \mathrm{nT}$ for both the PMN-PT and PZN-PT based laminates, which are about 1.7 times higher than the $1 \mathrm{nT}$ for PZT based ones. This increase in sensitivity originates from the increase in $\alpha_{\mathrm{ME}}$ for PMN-PT and PZN-PT laminates shown in Fig. 1. Panel (e) shows an example noise level from the low noise circuit in 


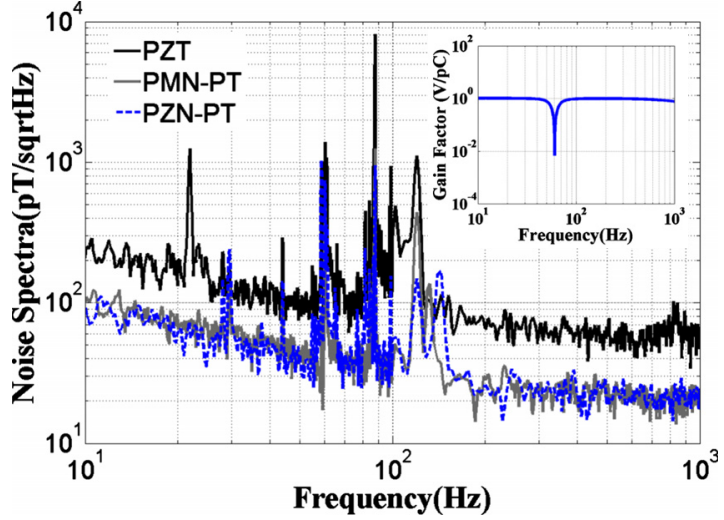

FIG. 3. (Color online) Noise spectra for the PZT, PMN-PT, and PZN-PT laminates with wide band circuit. Inset shows the wide band circuitry gain factor as the function of the frequency.

real time for zero ac magnetic field. One can see that the peak-to-peak noise level is about $5 \mathrm{mV}$ yielding a signal-tonoise ratio above 2 , which again has been kept constant during the sensitivity measurements for the various laminates.

Finally, the noise floors for the three ME laminate sensor units were measured in the frequency range of $10<f$ $<10^{3} \mathrm{~Hz}$. Over this frequency range, the gain factor (volt per pico coulomb) for our wide band detection circuit was shown in the inset of Fig. 3. We then obtained the following sensor transfer function, by which to convert the noise floor in $\mathrm{V} / \sqrt{\mathrm{Hz}}$ to that in $\mathrm{T} / \sqrt{\mathrm{Hz}}$ using the gain:

$$
\begin{aligned}
& \text { Conversion factor }(\mathrm{V} / \mathrm{T})=\frac{\alpha_{\mathrm{ME}}\left(\mathrm{pC} / 10^{-4} \mathrm{~T}\right)}{\text { Gain of } \operatorname{amplifier}(\mathrm{pC} / \mathrm{V})}, \\
& \text { Noise floor }(\mathrm{T} / \sqrt{\mathrm{Hz}})=\frac{\text { Noise floor }(\mathrm{V} / \sqrt{\mathrm{Hz}})}{\text { Conversion factor }}
\end{aligned}
$$

Please note that, we included a $60 \mathrm{~Hz}$ notch filter in the circuit to reject $60 \mathrm{~Hz}$ electromagnetic induction. In order to obtain the real noise floor for the ME sensor units, rather than that of the environment, the sensor units were tested in a mu-metal chamber without any signal input.

Figure 3 shows the noise floor spectra for all three sensor units. From this figure, we can observe that the noise floors for the Metglas-PMN-PT and Metglas-PZN-PT laminates are about $60 \mathrm{pT} / \sqrt{\mathrm{Hz}}$ in the frequency range of $f=10-100 \mathrm{~Hz}$ and about $20 \mathrm{pT} / \sqrt{\mathrm{Hz}}$ in the range of $f=200-1000 \mathrm{~Hz}$. This was much lower than that for the Metglas-PZT laminate which is $150 \mathrm{pT} / \sqrt{\mathrm{Hz}}$ and $70 \mathrm{pT} / \sqrt{\mathrm{Hz}}$ in the low and high frequency range, respectively. The reduction in the noise floor resulted from the increase in $\alpha_{\mathrm{ME}}$ for the laminates that had single crystal fibers, due to the much larger piezoelectric $d_{33}$ coefficient for PMN-PT and PZN-PT relative to PZT. The inset of Fig. 3 shows the gain factor (output/input) of amplifier which is calculated by using output voltage (volt) divided by input charge of ME sensor (pico coulomb) at frequency range from 10 to $1000 \mathrm{~Hz}$. It can be clearly observed that the notch filter part has pretty low gain factor that can reject $60 \mathrm{~Hz}$ electromagnetic interference (EMI) noise.

Our findings show that the choice for piezoelectric single crystal fibers with higher piezoelectric constants increases the magnitude of the ME voltage coefficient $\alpha_{\mathrm{ME}}$, resulting in a lower sensor magnetic noise. Thus, the magnitude of the noise floor scales with $\alpha_{\mathrm{ME}}$, where its spectral characteristics and dominate source are left unchanged for the three of ME laminates that we studied.

\section{CONCLUSIONS}

In summary, single crystal PMN-PT and PZN-PT fiber laminates were found to have a higher magnetic-field sensitivity (1.7 times) than Metglas-PZT ones. This was due to the fact that the ME voltage coefficient was 2.8 times larger. Studies of the noise power spectra revealed a reduction in the noise floor by a factor of $3-4$ times $(\approx 20 \mathrm{pT} / \sqrt{\mathrm{Hz}})$ for PMN-PT and PZN-PT laminates relative to PZT fiber ones.

\section{ACKNOWLEDGMENTS}

The authors gratefully acknowledge the help of Keith Malaughlin and Greg Beall from Science Applications International Corporation (SAIC). The authors thank the Department of Advanced Research Project Agency and the Office of Naval Research for support of this investigation.

${ }^{1}$ M. Fiebig, J. Phys. D 38, R123 (2005).

${ }^{2}$ G. T. Rado and V. J. Folen, Phys. Rev. Lett. 7, 310 (1961).

${ }^{3}$ J. Das, Y. Song, N. Mo, P. Krivosik, and C. E. Patton, Adv. Mater. 21, 2045 (2009).

${ }^{4}$ L. Yan, Z. Xing, Z. Wang, T. Wang, G. Lei, J. F. Li, and D. Viehland, Appl. Phys. Lett. 94, 192902 (2009).

${ }^{5}$ C.-W. Nan, M. I. Bichurin, S. Dong, D. Viehland, and G. Srinivasan, J. Appl. Phys. 103, 031101 (2008).

${ }^{6}$ J. Zhai, Z. Xing, S. X. Dong, J. F. Li, and D. Viehland, J. Am. Ceram. Soc. 91, 351 (2008).

${ }^{7}$ S. X. Dong, J. Y. Zhai, J.-F. Li, and D. Viehland, Appl. Phys. Lett. 89, 252904 (2006).

${ }^{8}$ Z. Xing, J. F. Li, and D. Viehland, Appl. Phys. Lett. 91, 182902 (2007).

${ }^{9}$ J. Das, J. Gao, Z. Xing, J. F. Li, and D. Viehland, Appl. Phys. Lett. 95, 092501 (2009).

${ }^{10}$ S. X. Dong, J.-F. Li, and D. Viehland, Appl. Phys. Lett. 83, 2265 (2003).

${ }^{11}$ P. Eitenne, G. Creuzet, A. Friederich, and J. Chazelas, Phys. Rev. Lett. 61, 2472 (1988).

${ }^{12}$ K. Shirae, IEEE Trans. Magn. 20, 1299 (1984).

${ }^{13}$ H. Barthelmess, M. Halverscheid, B. Schiefenhovel, E. Heim, M. Schilling, and R. Zimmermann, IEEE Trans. Appl. Supercond. 11, 657 (2001).

${ }^{14}$ J. Zhai, S. X. Dong, Z. Xing, J. F. Li, and D. Viehland, Appl. Phys. Lett. 91, 123513 (2007).

${ }^{15}$ Z. P. Xing, J. Y. Zhai, S. X. Dong, J. F. Li, D. Viehland, and W. G. Odendaal, Meas. Sci. Technol. 19, 015206 (2008). 